Carlos Orlando Fino Gómez*

\title{
Felipe Santiago Gutiérrez visita Bogotá por invitación de Rafael Pombo para fundar la academia Vázquez: una teoría femenina de la producción artística como producto del diálogo interatlántico ${ }^{1}$
}

Felipe Santiago Gutiérrez visits Bogotá accepting Rafael Pombo's invitation to found the Vázquez academy: a feminine theory of artistic production as a product of inter-atlantic dialogue

Cómo citar:

Fino Gómez, C. O. (2017). Felipe Santiago Gutiérrez visita Bogotá por invitación de Rafael Pombo para fundar la academia Vázquez: una teoría femenina de la producción artística como producto del diálogo interatlántico. Designia, 5(1), 9-29.

${ }^{1}$ Artículo mención de honor en el Premio Nacional de Crítica 2017, Ministerio de Cultura de Colombia.

*Profesional en Estudios Literarios (2010). Magíster en Historia y Teoría del Arte, la Arquitectura y la Ciudad (2012), con tesis laureada de la Universidad Nacional de Colombia. Candidato a doctor en Arte y Arquitectura. Ganó el Premio de Periodismo Cultural y Crítica para las Artes IDARTES (2012). Recibió el premio Casa de las Américas

en Ensayo artístico-literario (2014) por su obra José Lezama Lima: estética e historiografía del arte en su obra crítica; este libro fue además merecedor de la Mención de Honor del Premio Roggiano a la crítica del Instituto Internacional de Literatura Iberoamericana de la Universidad de Pittsburgh, entregado en Jena (Alemania)-2016; es ganador de la Maratón de Escritores Rock al Parque 2014. Es ganador de la Beca Distrital para Proyectos de Investigación en Literatura IDARTES, 2016. Recibió el reconocimiento de los 50 años de la Facultad de Ciencias Humanas, de la Universidad Nacional. Recibió mención de honor en el Premio Nacional de Crítica, Ministerio de Cultura, en 2017. carlos_fino@hotmail.com orcid.org/0000-0002-3883-3142
Palabras clave:

Rafael Pombo, Felipe Santiago

Gutiérrez, estética latinoamericana, modernismo, Academia Vásquez.

Key words:

Rafael Pombo, Felipe Santiago

Gutiérrez, Latin American aesthetics, Modernism, Vásquez Academy.

Recibido: 19/11/2017 Aceptado: 18/12/2017 
María apreciada:

El sábado nueve de la entrante semana, marcharé para Colombia donde me espera mi grande amigo Rafael Pombo, uno de los primeros poetas de la Nueva Granada, para hacerme cargo de la Academia Nacional de Pintura que el Gobierno de aquella República va a instituir por primera vez en ella [...] acogí contento la proposición que en una carta larga y expresiva me hace Pombo de que me vaya a poner al frente de la Academia que se va a abrir en su país; pues además de excitarme de todas maneras para que acepte, inclusive la de nuestra gran amistad, ha invocado mi patriotismo. [...] Adiós, pásalo perfectamente. 
El presente texto investiga la relación imagen-palabra como productora de argumentos estéticos entre el escritor colombiano Rafael Pombo y el pintor mexicano Felipe Santiago Gutiérrez, cuando este último visita Bogotá entre 1873 y 1875 con la misión de fundar la Academia Vásquez. La teoría femenina de la producción artística de Pombo se sustenta en dos hechos históricos: la fundación de una academia para mujeres por parte de Gutiérrez, primera de este carácter en Colombia; y la obra artística del mexicano, que de un lado se alimentó del naciente impresionismo europeo, pero de otro, por una conciencia latinoamericanista que coincidía con las posturas martianas visibles desde 1875, tal como se sintetiza en un par de ejemplos, el "Retrato de José María Espinosa" y "La Cazadora de Los Andes", que aquí son estudiados. La investigación se dedica a reconstruir esta contrapunteada línea argumental. Se busca demostrar que dicha teoría emerge del diálogo imagen-texto, texto-acción artística (la fundación de la Academia para mujeres), y alimenta la discusión estética sobre la relación hombre-naturaleza que, en este caso particular, surge como propuesta incluida en la búsqueda de un orden homeopático de mundo en la conciencia de la fundación de las repúblicas americanas.

\section{Abstract:}

This article analyses how the link between image and word was a basis for the production of aesthetic arguments between the Colombian writer Rafael Pombo and the Mexican painter Felipe Santiago Gutiérrez. This was the case when the later visited Bogotá between 1873 and 1875 in order to found the Vásquez Academy. Pombo's feminine theory of artistic production is grounded on two historical facts: the foundation of the first Colombian female academy by Gutiérrez, and the painter's artistic work, which drawn upon both the emerging European impressionism and a Latin American awareness coinciding with what recognizable in José Marti's thought since 1875. These two sources are reflected in a couple of examples studied here ("Portrait of José María Espinoza" and "The Hunter of the Andes"). The present work aims to demonstrate that the aforementioned theory emerged from the dialogue image-text and text-artistic creation, feeding the aesthetic discussion on the relationship between humans and nature. In the particular case considered here, this association arose as a proposal related to the search for an order of the world in the foundational ideas of the American republics. 
El 21 septiembre de 1873, Felipe Santiago Gutiérrez arribó a Bogotá en donde lo esperaba su amigo Rafael Pombo, tal como este lo comenta en un artículo aparecido en dicha fecha en el periódico La América. Gutiérrez llegaba con la misión expresa de fundar la Academia Vázquez de Bellas Artes, para lo cual Pombo tenía adelantadas gestiones, entre estas la redacción de la Ley que presentó Sergio Arboleda al Congreso de la República el 29 de abril de $1873^{2}$. Junto a otros temas de orden logístico y administrativo, la Ley establecía que la Academia se llamaría Vázquez en honor al pintor nacional Gregorio Vázquez de Arce Ceballos 3 . En el artículo "Informe sobre Bellas Artes”, publicado en La Escuela Normal el 7 de junio de 1873 (p. 184), Arboleda sostiene que Colombia se encuentra atrasada ${ }^{4}$ en cuanto a la creación de la Academia:

${ }^{2}$ En el número 127 de La Escuela Normal, publicado en Bogotá el 7 de junio de 1873, se puede leer:

El Poder Ejecutivo de Colombia acaba de sancionar la ley que crea la Academia Vázquez, que insertamos a continuación, junto con el informe del senador Arboleda que propuso su forma definitiva. Las últimas administraciones han consagrado especial atención y dado grande impulso a la causa de la instrucción pública; y ya era tiempo de concretar ese movimiento fomentando el cultivo de aquellos ramos que por unánime acuerdo forman la corona más gloriosa de la civilización de un país - el cultivo de lo bello, relacionado íntimamente con lo bueno y con lo verdadero- (...) Felicitamos al Congreso de 1873 y a la Nación por este gran progreso y cultura; y cómo suponemos que, para cada ramo, bajo los cuales se abrirán clases diarias, públicas y gratuitas en locales adecuados, nos aventuramos a predecir que antes de diez años los talentos artísticos que poseemos estarán fructificando. (Creación de la Academia Vásquez, 1873, junio 7, p. 183).

${ }^{3}$ Además, se establece que contará con cinco escuelas (pintura, grabado, música, arquitectura y escultura); que cada escuela será dirigida por un profesor, con asignación anual de \$3000; que tendrá biblioteca y archivo de Bellas Artes; que tendrá un museo, en el cual se recogerán y conservarán las obras artísticas de la Nación; que se contratará a cinco artistas de reconocida trayectoria para dirigir las escuelas; que los profesores deberán cuidar el ornato de los edificios nacionales; que se donará un edificio en la capital a la Academia; y que se dará un monto de \$18.000 iniciales para gastos dotacionales. El proyecto es firmado el 4 de junio de 1873 por José María Quijano Otero, secretario de la Cámara; por M. Plata Azuero, presidente plenipotenciario del Senado; por José María Maldonado, presidente de la Cámara; y es refrendado por el presidente Manuel Murillo Toro.

${ }^{4}$ Efectivamente, Colombia vivía un retraso ya que el proyecto de la academia data de 1873 pero solo hasta 1886 se oficializa, no bajo la gestión de Pombo y la dirección de Gutiérrez sino de uno de sus estudiantes, el exguerrillero conservador y dibujante Alberto Urdaneta. La fecha es tardía ya que, en México, San Carlos se funda en 1781, la Academia en Guatemala en 1797, en Argentina en 1799 , en Perú en 1816 y en Cuba en 1818. En el informe, Arboleda sostiene que el desarrollo material sin el moral es catastrófico para un pueblo y que las artes juegan un papel preponderante en esta empresa. 
Este proyecto de ley tiende ciertamente a llenar un notable vacío que se observa en nuestro país y no en otro país culto, exceptuando probablemente el Paraguay, Bolivia y dos o tres estados centroamericanos. Vuestra Comisión tiene noticia de que en todas las demás secciones de América se ha hecho algo para fomentar el cultivo de las Bellas Artes, rasgo hoy característico de los pueblos civilizados.

La llegada de Gutiérrez parecía ser lo que faltaba para que se abriera la Academia, pero los recursos no llegaban y en noviembre de aquel año aún no se había iniciado la ejecución del proyecto, que de manera oficial jamás inició5. En "Memorial sobre la Academia Vázquez", publicado en La América el 20 noviembre 1873, se recuerda al gobierno la llegada de Gutiérrez con una carta colectiva liderada por Rafael Pombo:

Ciudadano Presidente de la República: Señor. Los abajo firmados, pintores de profesión aficionados a ese arte sabiendo que ya se encuentra en esta capital el afamado artista mexicano señor Felipe Santiago Gutiérrez, y constatándonos por los diversos trabajos que hemos visto de él, y por su larga y completa educación en el arte, muy competente para dirigir una escuela de pintura, la cual daría a este ramo un impulso extraordinario en Colombia, nos dirigimos a vos respetuosamente solicitando que dispongáis se abra sin demora bajo su dirección esa parte de la Academia Vázquez, decretada por el último Congreso. (Pombo, 1873, p. 1).

Ante la indiferencia del gobierno pero el apoyo de Pombo, Felipe Santiago Gutiérrez fundó dos academias privadas en las cuales se dedicó a la enseñanza y organizó exposiciones, conjuntamente con sus estudiantes y artistas de trayectoria ${ }^{6}$. Antes de analizar su accionar pedagógico vale la pena acercarse a dos obras, el retrato realizado a José María Espinosa y el ejercicio libre titulado “La Cazadora de Los Andes”,

${ }^{5}$ Son varias las razones por las cuales no se abrió la Academia Vázquez. La primera hipótesis es que se trataba de un proyecto liderado por conservadores opositores reunidos alrededor del periódico La América, quienes criticaban continuamente al gobierno radical liberal de Manuel Murillo Toro. El presidente de la unión no mostró mayor simpatía por el proyecto y relegó su importancia ante otros, como la deuda externa o el Ferrocarril del Norte, asunto discutido a diario en los periódicos del momento. Al respecto, Álvaro Medina (2014) sostiene:

¿pero no lo permitía la situación económica del país? Según el historiador Paul McGreevey, la balanza comercial con los Estados Unidos era desfavorable a Colombia y el acumulado pasó de 61.8 millones de dólares en 1872, a 82.8 en 1873 y alcanzó los 92.9 millones en 1874, desajuste negativo que solo en 1875 empezó a declinar. Se concluye que a la falta de voluntad política se sumó la incapacidad económica y por eso la Academia no fue nunca una realidad, razón por la cual los interesados en aprovechar la presencia de Gutiérrez se reunieron para crear una academia privada (Medina, 2014, p. 91).

${ }^{6}$ Se destacan la individual que realizó en la Casa de las Secretarias, en donde exhibió medio centenar de piezas, y la colectiva que tuvo gran acogida y alcanzó el número, nunca antes visto, de 400 obras, contando las del mexicano y los aportes nacionales. 
en relación con el lenguaje suelto que alude a las innovaciones pictóricas, las cuales resultan análogas a las que tuvo el modernismo literario. Esta hipótesis se traza gracias a la cita realizada por Álvaro Medina del historiador Manuel Caballero-Banard en Procesos del arte en Colombia, en la cual concluye los aportes que realizaron los viajes trasatlánticos en la producción y pensamiento de Felipe Santiago Gutiérrez, y de este modo se sostiene que la academia bogotana nace, de manera privada, con elementos que hacen parte de una combinación entre la academia neoclásica, aprendida por Gutiérrez con Pelegrín Clavé y Eugenio Landesio, y al mismo tiempo con métodos pictóricos que muestran una expresión suelta, enriquecida y propia de precursores del impresionismo, como son Eduardo Rosales, Fortuny, Madrazo y Courbet ${ }^{7}$.

${ }^{7}$ Medina (2014) cita a Manuel Caballero-Banard quien sostiene que Felipe Santiago Gutiérrez,

Realiza su sueño de llegar a París el 10 agosto de 1868. Durante un mes y días admira las obras expuestas en los museos, así como la gran metrópolis, donde más tarde triunfará y se convertirá en el primer mexicano que logre una presea de arte europeo. De allí se traslada a Roma donde ocurrirán tres hechos que marcarán en forma permanente su vida artística; el primero, será la gran decepción que recibe al inscribirse en la Academia de San Lucas, por ese entonces considerada el templo mayor de las artes en todo el mundo, y descubrir su arcaico y obsoleto sistema de enseñanza, lo cual lo decide a establecer su estudio particular. El segundo, al enfrentarse por primera vez al desnudo femenino, impresión que aprovechará más tarde en Suramérica, con su cuadro "La Amazona de Los Andes". Esta obra causó un gran escándalo al ser presentada la exposición anual de la Academia de San Carlos, en México. Esto se explica por haber sido el primer desnudo femenino integral que presenta la pintura mexicana del siglo XIX. Para poder ser exhibido, las autoridades de la Academia ordenaron que fuera colgado en el gabinete dedicado a los pintores extranjeros y la pintura europea; el tercer punto trascendental de su estadía en Roma fue conocer al crítico de arte francés Castagnary, amigo y propagandista incondicional de Gustavo Courbet, el cual lo convenció de que se trasladara a París. Su residencia en dicho lugar fue de seis meses, tiempo en que convivió con los iniciadores del impresionismo en la Ciudad Luz, donde se venían gestando desde hacía algunos años las ideas innovadoras, la libertad de expresión y los conceptos de un nuevo arte (p. 85). 
Retrato de José María Espinosa y el dominio del lenguaje precursor del impresionismo

Gutiérrez retrató en dos oportunidades a José María Espinosa ${ }^{8}$, el pintor famoso por ser el abanderado del ejército de Antonio Nariño en las luchas independentistas.

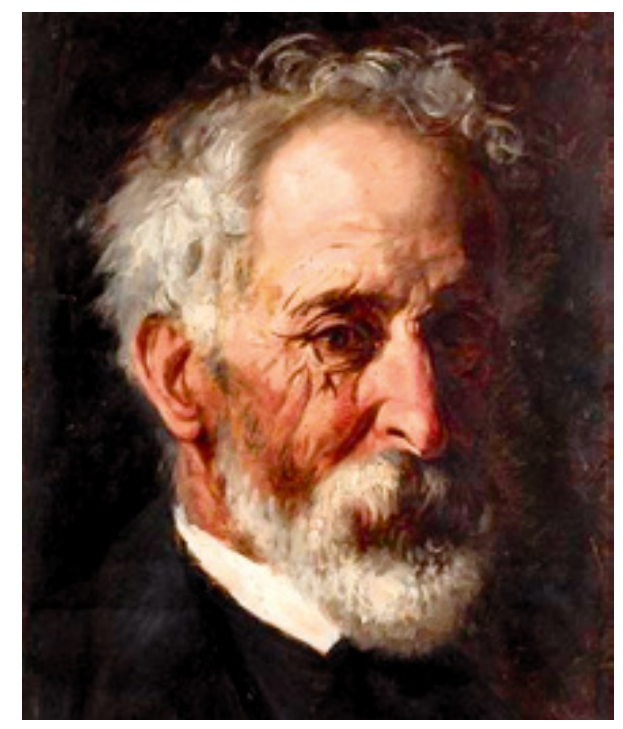

Figura 1. Retrato de José María Espinosa. Felipe Santiago Gutiérrez, 1874. Fuente: Tomada del Catálogo del Museo Nacional de Colombia. Reg. 2251.

En el número 187 de La América, publicado el 11 de mayo de 1874 y con el seudónimo de Florencio, Rafael Pombo publica el artículo "Exposición Gutiérrez", en el cual señala:

El que sepa, por ejemplo, cuan raro y difícil es dar tono de vida y transparencia a las carnes, y determinar distintamente las telas, y cuantos pintores de nota han luchado en vano por hacer trajes blancos que causen ilusión perfecta (...) Hay allí prodigios de carnación, como el San Jerónimo; prodigios de escorzo, como un brazo de cierta señorita de blanco; prodigios de dibujo y actitud, como el retrato del señor Groot; prodigios de franqueza o sea decisión y vigor de ejecución, como el del señor Espinosa. (p. 2). 
Álvaro Medina (2014) sostiene en relación a este apartado que Gutiérrez puede considerarse como el pionero del retrato psicológico en Colombia:

\begin{abstract}
La exaltación que hizo de los brochazos del retrato de Espinosa, firmado por Gutiérrez, lo dice todo (...) El cuello de la camisa blanca restalla contra el fondo negro con un plano de delgadas pinceladas níveas de gusto abocetado que se yuxtaponen dejando fisuras sin cubrir, mostrándose reacio a cubrir la totalidad de la superficie de una forma pareja. La soltura del pincel es visible en la cabellera, zona en la que el pelo canoso tiene un tratamiento que acentúa el despeine del prócer y pintor para comunicar la sensación de personalidad recia. No se trataba de meros formalismos, ni de alarde técnico. El retrato psicológico acaba de hacer su aparición entre nosotros (p. 96).
\end{abstract}

En 1885 Felipe Santiago Gutiérrez presenta su Tratado del dibujo y la pintura: con un apéndice de los diversos caracteres de las escuelas antiguas y modernas. En la introducción señala que "la pintura es la representación o reproducción de todos los objetos que vemos en la naturaleza" (p. 1). Más adelante agrega, y es tal vez la confirmación de lo que Álvaro Medina denomina retrato psicológico, que:

\begin{abstract}
La pintura no sólo tiene la misión de imitar la forma externa de los objetos; sino que reproduce también con energía el alma de la naturaleza y las pasiones de los individuos de la especie humana; habla así mismo el lenguaje del corazón y engendra sensaciones agradables o tristes de amor y odio, de grandeza y entusiasmo (...) en una palabra es una segunda creación. (p. 1).
\end{abstract}

La primera definición de Gutiérrez pertenece a una poética clásica y neoclásica, la del arte como representación. Pero de inmediato relativiza esta opinión: el arte es creación. Además de encontrarse entre los dos lindes, añade que la pintura no se debe quedar pintando solo las superficies, sino también lo que no ve, como las pasiones y emociones.

\footnotetext{
9 Es preciso anotar que durante el periodo en que dictó clases en la Academia San Carlos, Pelegrín Clavé escribió unas Lecciones de Estética de las que en 1990 la Universidad Nacional Autónoma de México publicó una edición facsimilar. En esta obra se deja ver la inclinación nazarena del artista, la cual intentó recuperar aspectos medievales y renacentistas para la pintura, tales como la perspectiva, la composición sagrada, el preciosismo en el color y un especial tratamiento sobre los temas bíblicos y los detalles. Sin embargo, Felipe Santiago Gutiérrez y Santiago Rebull, ambos discípulos de la Academia San Carlos y estudiantes de Clavé, trataron temas de la historia sagrada y civil del cristianismo con el ánimo de marcar posiciones en su contexto político, como en el caso de "El juramento de Bruto", obra de Gutiérrez de 1857. En este pintor se nota una transición de las ideas estéticas aprendidas de su maestro catalán, quien realizó un periplo que lo llevó a un sinnúmero de viajes, de los cuales Bogotá fue una importante escala.
} 


\section{Retorno de Gutiérrez a México: la confirmación martiana}

En 1875 Felipe Santiago Gutiérrez retorna a México ${ }^{10}$. La Revista Universal de ese país reproduce el 3 de junio de aquel año un texto escrito en Bogotá el 21 de marzo, autoría de Rafael Pombo, en el cual se indica el aporte del pintor mexicano a la historia de la didaxis artística en dicha ciudad ${ }^{11}$. José Martí también escribe sobre su obra. En el artículo titulado "Felipe Gutiérrez", publicado en la misma revista el 24 de agosto de 1875 (p. 90), Martí (2000b) destaca su respeto a la tradición y a la vez la innovación a partir del estudio de los pintores clásicos. De manera ecuménica, Martí enumera la Madrid referencial del pintor mexicano y denota en él esa reunión de temporalidades:

Felipe Gutiérrez pinta con grandes rasgos entre grandes sombras. No diluye la luz: la descompone y la contrasta; no dibuja con líneas, sino con experimentados golpes de pincel. No hay en él claroscuro; hay en él claro y oscuro: un claro luminoso y atrevido; un oscuro lleno de potencia y de vigor. Opone el uno al otro: no los concilia. Es el estilo libre y propio de un pintor que ha visto la vida en los cuadros de Miguel Ángel, Ribera y Tintoretto. Gutiérrez pinta pronto, pinta mucho y pinta muy bien. Hay en él algo de la imponente frialdad de Rosales. El artista español pintaba, más que con colores, con músculos y nervios. Gutiérrez anda aprisa por ese camino.

\footnotetext{
${ }^{10}$ En una nota titulada "El señor D. Felipe Santiago Gutiérrez" de la revista Universal de México del 30 de mayo de 1875, José Martí da la noticia e informa que el artista viene de tierras bogotanas, donde permaneció entre 1873 y 1875 tras ser convencido por Rafael Pombo de fundar allí la Academia de pintura:
}

Este nombre debe ser muy conocido para nuestros lectores; está ligado con nuestras glorias nacionales; es en nombre del eminente pintor que, después de obtener merecidos laureles en Roma y en París, fue llamado a la dirección de la Academia de Bogotá donde ha permanecido hasta el mes de marzo último. El último vapor americano atracó en las playas de su patria, y antenoche llegó a la capital. El señor licenciado Sánchez Solís, uno de sus admiradores más entusiastas y de sus más decididos protectores, le obsequió esa misma noche, en su espléndida casa, con un magnífico buffet, al que asistieron algunos de nuestros más notables artistas y entre ellos los señores Pina y Obregón (Martí, 2000a, p. 171).

${ }^{11}$ Pombo (1875, junio 3) afirma en dicho texto:

Desde la muerte de Gregorio Vázquez hasta la venida de Felipe Gutiérrez, ha habido un blanco de siglo y medio en este país en materia de pintura (...) Gutiérrez se dedicó a enseñar gratuitamente, deshizo el mal gusto con su poderoso pincel, y aún nos deja noventa retratos y muchos cuadros y estudios, suficientes muestras de la grande escuela española, para que en adelante no admiremos lo que no tenga mérito ninguno, y exijamos de todo pintor una destreza y una seguridad, fuerza y verdad, que en muchísimos años ningún artista de los que venga aquí, podrá presentarnos. (p. 2). 
Martí tiene en sus ojos las recientes polémicas que despertó en Madrid Eduardo Rosales Martínez, quien trataba grandes temas históricos desde una fuerza grandilocuente y privada, haciendo gala de gran libertad y dominio técnico, muy emocional, de una lectura gruesa en la cual el lienzo es frecuentemente golpeado por el pincel. Esta comparación revela a un Gutiérrez de "grandes rasgos entre grandes sombras" como lo es Rosales, pero también con un contagio emocional interno, muscular, nervioso sobre el lienzo. Ambos pintores tienen rasgos profusos de la tradición hispánica: los críticos refieren un velazquismo de Rosales y Martí lo compara con El Españoleto (1591-1652), así como con la tradición del fresquismo italiano y la pintura del veneciano Tintoretto (1518-1594).

Luego de estudiar el retrato del licenciado Juan Gómez, gobernador de Puebla ${ }^{12}$, José Martí insiste en que la innovación de Gutiérrez tiene que ver con el lenguaje, uno que encuentra no para embellecer la realidad, sino para transmitir y comunicar emociones. Así lo expresa en el ya referido artículo del 24 de agosto de 1875 en la Revista Universal:

\footnotetext{
No hace líneas: hace rasgos. Es un pintor de gran manera: no complacerá en todos los gustos. Lo que Gutiérrez pinta, satisface en algo la aspiración humana a la grandeza. Es más hermoso que bello. No hará nunca un cuadro lindo; pero hará cuando quiera un cuadro grande y sorprendente (Martí, 2000b, p. 91).
}

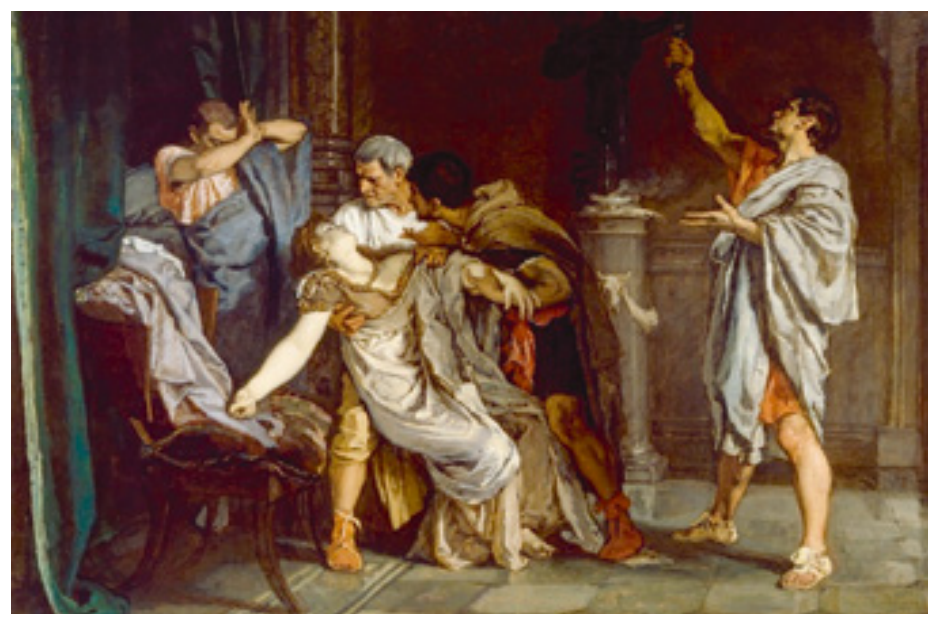

Figura 2. La muerte de Lucrecia. Eduardo Rosales Martínez, 1873. Fuente: Catálogo del Museo del Prado. Número de Catálogo: P04613. 
Para ambos - Rosales y Gutiérrez - la prioridad pictórica insta en la "impresión" general del cuadro: más en su composición que en los detalles, propios de una pincelada académica y preciosista. Francisco Calvo Serraller (1990) comenta la polémica desatada por "La muerte de Lucrecia" (1873):

\begin{abstract}
El sentimiento de injusticia que estos críticos produjeron en Rosales fue, sobre todo, por la incomprensión que demostraban respecto a sus verdaderos propósitos artísticos. "Al decir de la mayoría - escribió en su descargo el propio Rosales -, mi cuadro es un cuadro no concluido y por tanto una obra defectuosa y digna de censura; convengan parte en ello, pero no en todo, el cuadro no está terminado, pero el cuadro está hecho (...) Una obra del carácter de la presentada por mí, no es un cuadro de gabinete, que ha de ser primorosamente ejecutado para que se seduzca la vista, es una obra de impresión y de impresión vigorosa y enérgica, que debe ante todo hablar al alma y no al sentido (...) mi cuadro es un cuadro de impresión, la escena es eminentemente dramática, mi mayor anhelo era que el cuadro hiciera estremecer desde el primer momento (...) de ahí la vehemencia en el hacer, la ejecución desaliñada y brutal; yo veo que con una ejecución primorosa el cuadro no sería lo que debería ser." (p. 56).
\end{abstract}

En este párrafo se explican varios aspectos que no solo tienen que ver con la forma de ser del artista moderno o con el lenguaje moderno de Rosales y Gutiérrez, sino con la forma de ser modernista de Martí. En los tres casos se opta por una nueva configuración artística con un lenguaje suelto, guiado por la impresión de las cosas sobre nosotros y no por una captura realista o idealizada de la realidad. Por ello, el subjetivismo y la franqueza. Si bien no es posible sostener que alguno de los tres sea impresionista, pues tampoco es su intención alinearse con el movimiento francés, sí hay un tópico conceptual que alimenta sus obras: fijarse más en la impresión que en el detalle; calcular el foco de la pintura o del texto y bocetarlo; pero, sobre todo, ejecutarlo con carácter y fuerza física.

Hay una especie de manifiesto plástico en el que se quiere o desea abandonar la normatividad académica y se busca una decidida experimentación que recupere la singularidad y el impulso creativo corporal en la obra, en la praxis artística. Pero, en los tres casos, la disputa es con la normalización académica que deviene en preciosismo, y no con la tradición pictórica. Aquí se encuentran las claves de la manera castellana o la vertiente castellana de ser modernista de José Martí: es clave recordar que en 1875 Martí es un ciudadano español nacido en ultramar, exiliado y con deseos independentistas. Pero él desea alcanzar la emancipación de España también en el seno de lo español, siguiendo entre otros a Cervantes y Quevedo, así que traza vínculos claros con el Siglo de Oro para encontrar una manera de arraigarse en las 
necesidades de la actualidad: una forma de impresionar a través de una lengua suelta que bocete escenas con agilidad. Teóricamente, la apuesta verbal de Martí se puede catalogar como una écfrasis de la impresión, o esa capacidad de recrear una escena con un pequeño conjunto de palabras.

\section{“La cazadora de Los Andes": manifiesto visual de la teoría femenina del arte}

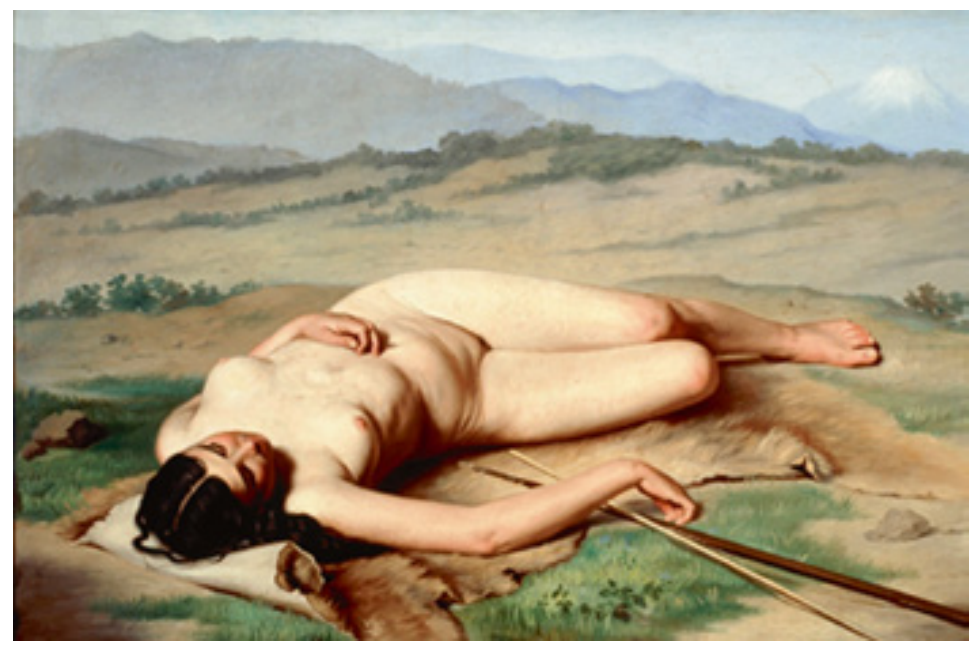

Figura 3. La Cazadora de Los Andes. Felipe Santiago Gutiérrez, 1873-4. Fuente: Tomada del Catálogo del Museo Nacional de Arte de México.

En su visita a Bogotá, Felipe Santiago Gutiérrez ejecutó el óleo sobre tela fechado en 1874 con dimensiones de 90.5 por $146 \mathrm{~cm}$, titulado la "Cazadora de Los Andes". Un brevísimo análisis iconográfico permite ver el tema, ya revolucionario para la pintura en América Latina, por ser el primer desnudo íntegro al óleo de un autor mexicano, y además fue pintado en Bogotá ${ }^{13}$. Gutiérrez pinta una mujer desnuda, como si fuese una Diana cazadora que atraviesa el cuadro de la parte inferior izquierda en donde ubica la cabeza, y se extiende hacia la parte superior derecha.

\footnotetext{
${ }^{13}$ Para Bogotá es la primera vez que se pinta un desnudo del original, y aunque no se exhibe en la capital, si se tiene noticia de él gracias a un poema que le dedica Rafael Pombo. Probablemente es uno de los primeros intertextos de Epifanio Garay, estudiante de Gutiérrez, en su cuadro "La mujer del Levita", de 1899, considerado como el primer desnudo exhibido en Colombia por un autor colombiano, a sabiendas de que Epifanio Garay nació en Panamá.
} 
La cazadora descansa probablemente luego de una jornada de labores sobre una pelliza, que sugiere la referencia hercúlea y amazónica. A su lado trae arco y flecha. La figura aparece focalizada en la cabeza y pierde el foco en los pies. Una poderosa distinción entre figura y fondo da la impresión de que la cazadora flota sobre Los Andes bogotanos. El fondo está sumamente cuidado y se trabaja con pinceladas gruesas y menor carga y nitidez en los colores que la cazadora, quien ingresa al paisaje de modo secante, pareciendo un río flotante sobre él. El cuerpo aparece escorzado y la mujer se encuentra en actitud contemplativa.

Su tópico es la indistinción entre el hombre y la naturaleza: Los Andes se dibujan con la misma sinuosidad de la cazadora que repite las ondulaciones de las montañas. Pero esta encadena la relación peso-liviandad, ya que, aunque representa la mátria telúrica, su cuerpo parece que flotara sobre Los Andes que se presentan vaporosos. La tierra y el aire son los elementos principales y revelados, que implícitamente guardan el agua en el río que traza la mujer de los pies a la cabeza y el fuego que está en reposo y se nota en el candor de su carrillo bermejo.

Eugenio Landesio y José María Velasco aparecen como intertextos visuales, ya que es preciso recordar la "Vista del Valle de México", pintura icónica del paisajismo mexicano autoría de Velasco en la que un aspecto primordial es su perspectiva, la cual produce una sensación aérea. El aire de esta pintura se siente o se ve gracias a la separación de vacío y profundidad que construye el punto de vista del espectador.

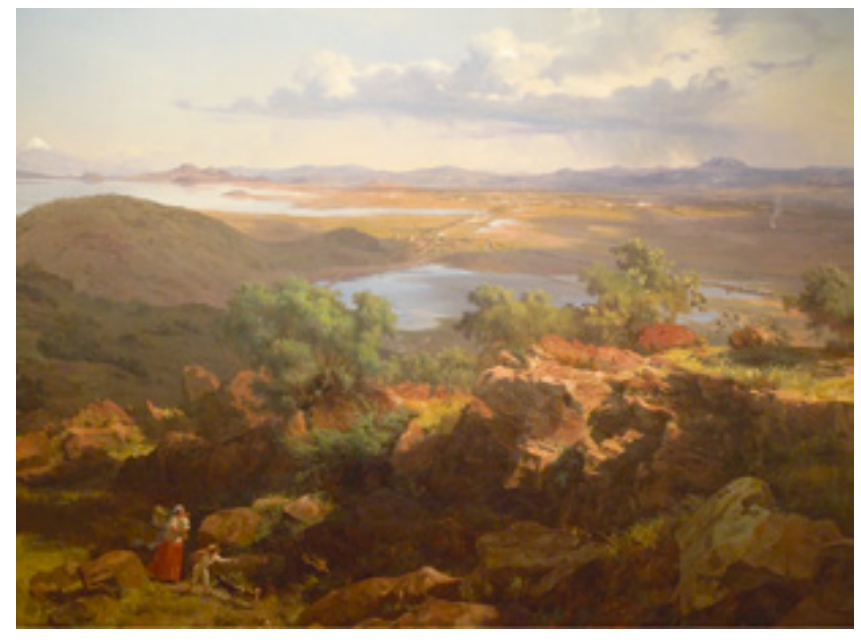

Figura 4. Vista del Valle de México. José María Velasco, 1875. Fuente: Tomada del Catálogo del Museo Nacional de Arte de México, Ciudad de México. 
En "La cazadora de los Andes", Gutiérrez exhibe gran uso de recursos compartidos con el modernismo literario: un lenguaje pictórico suelto en que la imagen artística da cuenta de la visión paradisiaca andina; la capacidad de hacer sensible un elemento transparente como es el viento gracias a la focalización y el desenfoque selectivo, reforzado por una perspectiva aérea que configura una poética de lo levitante y lo telúrico como elementos sagrados. Además, ese lenguaje moderno le hace conciliar con el nazarenismo al pintar una mano de la virgen a la cazadora, convirtiendo la escena en antediluviana y edénica, para conformar un lenguaje simbólico-alegórico que transmite el mensaje de la sacralidad del paisaje americano ${ }^{14}$.

\section{La Academia de mujeres de 1874 y la teoría de lo femenino en la producción artística}

Junto a la causa de la emancipación simbólica del continente como primer objetivo, el modernismo latinoamericano trabajó en pro de la emancipación gnoseológica y educativa de la mujer, tanto en áreas de la educación regular y normal como en la educación artística. Intelectuales como Eugenio María de Hostos, José Martí y Justo Sierra, entre varios otros, lucharon en aras de la educación científica de la mujer. Es preciso adjuntar a este grupo el nombre de Felipe Santiago Gutiérrez, para 1874 una figura descollante en el campo artístico, quien fundó una academia para

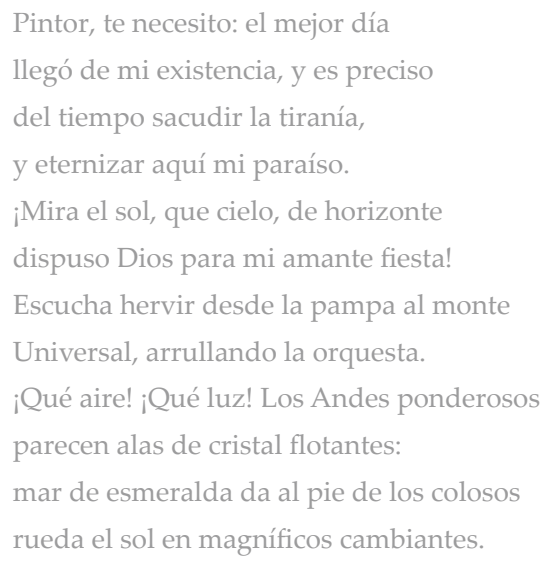


mujeres. Cabe destacar que el mexicano impartió los mismos temas y enseñó las mismas técnicas para los dos géneros. Este acto era sin duda revolucionario, no solo en una ciudad como Bogotá donde la academia no se había establecido, sino en el contexto latinoamericano, en el cual eran escasos los centros de enseñanza artística con programas académicos femeninos.

Vale la pena resaltar que dicha fundación, así como la participación de las estudiantes en la exposición de Bellas Artes que tuvo lugar en el antiguo local de las Secretarias en Bogotá en 1874, revierte y pone en juicio las afirmaciones realizadas por la investigadora Carmen María Jaramillo, quien afirma que antes de la Academia de Bellas Artes fundada por Urdaneta en 1886 no hubo educación artística para la mujer en una academia ${ }^{15}$. Pero es muy distinta la naturaleza de los hechos. En agosto de 1874, 12 años antes de la fundación de la Academia de Bellas Artes y en pleno Radicalismo Liberal, Pombo escribe "Exposición de Bellas Artes" para La América ${ }^{16}$. En dicho texto no solo informa los resultados de la academia para las mujeres fundada por Gutiérrez, sino que testifica la participación pública de sus estudiantes como artistas concursantes en la exposición ${ }^{17}$ :

\footnotetext{
${ }^{15}$ Entrevista realizada por Christopher Tibble y publicada en la revista Arcadia el 28 junio de 2016, en la cual se puede leer la siguiente afirmación:
}

\footnotetext{
-C. Tibble: Usted inicia en la Regeneración (1878-1898). ¿Por qué no antes? -C. M. Jaramillo: La Regeneración es un momento histórico muy contradictorio. Es conservador, pero también es el que moderniza al país. Es una modernidad conservadora, en la que lo público empieza a ser muy importante. Allí es cuando se empieza a dar mucha más importancia a la educación de las mujeres. En las clases altas, por ejemplo, había cierto acercamiento a las artes, pero siempre en la casa, en privado, en un ambiente controlado. En las clases estaban las mamás y las tías presentes con el profesor. Además, la Escuela de Bellas Artes abre en 1886, aunque exclusivamente para hombres. Es sólo a finales del diecinueve cuando se abre una cátedra de paisaje en la que pueden entrar mujeres, pero en un salón aparte. Y es sólo cuando llega a la dirección Andrés de Santamaría en 1904 que las mujeres pueden participar en la clase con modelo desnudo. Si las mujeres no podían pintar desnudos, ¿qué podían pintar? Pues bodegones, y así se volvieron pioneras en el país. (p. xx).
}

\footnotetext{
16“Exposición de Bellas Artes” se publicó en tres entregas en los números 206, 207 y 209 correspondientes a los días 4, 7 y 13 de agosto de 1874, respectivamente, del periódico La América de Bogotá.
}

\footnotetext{
${ }^{17}$ Uno de los puntos de innovación tiene que ver con la divulgación artística. En la exposición colectiva el interés publicitario se centró en la competencia por categorías y el salón de mujeres tuvo competencia propia. Esto es una prueba evidente de que el arte realizado por mujeres en el caso colombiano durante el siglo XIX no puede ser solo entendido para espacios privados, sino que en ciertos casos adquirió esa dimensión pública y participada. El salón y la exposición son maneras de avanzar en pro de la emancipación artística de la mujer. Pombo describe la experiencia al ingresar:
}

Entremos a esta sala, única en Colombia, por ser empapelada por mano de las Gracias mismas. Lo primero que encontramos a la izquierda son unos estudios de colorido, ya copias, ya originales, que se nos informa son primeros ensayos de sus autoras en 


\begin{abstract}
Al entrar en la tercera sala sentimos una emoción de extraordinario placer, y no podemos menos de bendecir el nombre de Felipe Santiago Gutiérrez, apóstol de beneficencia y de cultura entre nosotros. Sus paredes están colgadas, cubiertas con los trabajos de las señoras y señoritas de su Academia, y representan cuatro o cinco meses de horas robadas por la mano generosa del artista al fastidio (patrimonio de la mujer entre nosotros) y transformadas por él en deliciosa sociedad y en fraternal cultivo de la gracia, el espíritu y el sentimiento. Muy natural es que nos regocijemos con tal espectáculo, siendo como son nuestros sentimientos en este particular los expresados en el discurso en verso titulado "La educación es la fuerza de la mujer" (p. 1).
\end{abstract}

"La educación es la fuerza de la mujer" fue publicado el 28 de febrero de 1874 en La Escuela Normal. En tal discurso, Pombo planea que las arraigadas costumbres y la falta de medios civilizadores e instituciones para su beneficio no han colaborado en el proceso de emancipación de la mujer, y que más bien, su lugar apaciguado en el espacio doméstico no les permite ganar confianza y autonomía para dedicar sus horas a actividades que coadyuven al progreso del país. Para Pombo, el elemento que puede favorecer la transformación del papel doméstico y pasivo al público y activo de la mujer, es, con mayor prelación, su educación científica:

\footnotetext{
La mujer que al estudio se aficiona

y abrió al fin de su espíritu las alas

a admirar en sus dones y portentos

la omnipotente diestra soberana;

La que el universo la armonía

vio a la luz de la ciencia, y con la vasta

posesión de la historia el triste juego
}

\footnotetext{
este género. De ellos nos agradan principalmente las Plegarias de las señoritas Emilia Espinosa, Dolores Valenzuela y Josefina Barbieri. En la primera y tercera hay muy buen principio de color, y en la segunda bastante firmeza de ejecución y simpática expresión en la cabeza de la niña (...) Como son muchas, nos faltaría espacio para dar de cada una de ellas una idea circunstanciada; baste decir que en todas hay méritos relevantes, como son, la exactitud del parecido con los modelos, una modelación fina y excelente, una entonación verdadera, el más esmerado mecanismo, y tal suavidad y verdad, en todas, que no pueden menos que cautivar la atención. (Pombo, 1874, agosto 13, p. 1).
} 
del egoísmo y vanidad humana.
La que ha logrado de las Bellas Artes
sentir la magia enalteciente y casta
y penetrar en el santuario excelso
do el Sanzio pinta y Palestrina canta;
La que de una Stael apreciar supo
la crítica profunda y delicada
y de Rosa Bonheur los lienzos puros
virtud y poesía en el aire y vacas... (p. 64).

Para Pombo, la mujer necesita con urgencia emanciparse ya que tanto el contexto colombiano como el latinoamericano, que se encuentran en sus procesos de emancipación política y simbólica y en la fundación de sus instituciones nacionales, requieren la colaboración civil de todos sus ciudadanos. Igualmente, la educación científica, el liderato, la libertad y ciudadanía para la mujer están en la línea filosófica de América como un nuevo proyecto de humanidad. El poeta no solo reconoce en la mujer el lugar de la creación vital, relacionada con la creación artística, sino que la fuerza de lo femenino, tanto en su percepción sensible, como en la capacidad de engendrar lo vital, lo necesario y lo nuevo, son elementos fundamentales para la creación artística en ambos géneros. Dicho esto, Pombo (1874, agosto 13) continúa:

\begin{abstract}
La mujer es el precioso compendio de la Naturaleza, sensible a ella más que al hombre, y más llamada que él a vivir en dulce y fecunda comunión con ella. Hay muchos secretos de entre las dos, a los cuales es refractario el espíritu del hombre (...) El hombre, animal que mueve pleitos, que mata gente, que con tranquilidad de conciencia desola casas y provincias, que (como Fausto) corta árboles centenarios y quema los hogares de los ancianos que le embarazan la vista de la fábrica o del puerto (...) esta fiera tan rara, tan dañina y tan estúpida de corazón, no merece cultivar aquellos ramos semi-angélicos, tan superiores a él por lo rudo y frío de su percepción, por su propia gracia y elasticidad de espíritu, por su escasez de facultad simpática o asimiladora, y hasta por la constitucional tosquedad de sus manos. (p.1).
\end{abstract}

Las palabras de Pombo apuntan a una comprensión cultural relacionada con la hipótesis de que el predominio patriarcal llega con la edad de hierro, edad en que la estructura cultural se centraba en la guerra y el dominio de los territorios. Al iniciar esta, finaliza la edad que concentraba el poder en la fertilidad de la tierra, el conocimiento de la agricultura y las peticiones hacia las divinidades atmosféricas. El neolítico es un mundo en que la simpatía es la norma vital, en tanto aún existe un resquicio de la cosmovisión paleolítica en la que no existía mayor distinción 
entre el hombre y naturaleza. En el paleolítico el dominio femenino era total, pues la supervivencia humana dependía casi por completo de la capacidad fecundativa de la matriarca, en condiciones contextuales con presencia de megalodontes y una sociedad de cazadores (por no decir presas) y recolectores. Así, la apología a la mujer está asociada a la necesidad americana de replantear las relaciones entre hombre, naturaleza y cultura, con el ánimo de entablar los vínculos sagrados, la vida fecundativa y la providencia que comparten lo femenino y la naturaleza. Es también la solución para que el arte americano cree una nueva alianza que sea una alternativa a la hegemonía patriarcal del consumo, la desacralización de la naturaleza, la razón alopática y el dominio a través de las prácticas colonizadoras y bélicas. De esta manera, el arte americano realizado por las mujeres y el arte con criterios culturales femeninos puede colaborar en la construcción de un nuevo pacto simbólico cultural. En este, los valores naturales han de ser aquellos que retroalimenten la sociedad y la cultura, enmarcadas en un contexto natural. Pombo (1874, agosto 13) sostiene:

\section{No es extraño que el hombre haya hasta ahora repugnado tanto el abrir a la mujer estas vías de ejercicio y desarrollo, ya por lo mal que él las comprende y practica, sin sentirlas y embalsamarlas de amor, ya por la sospecha de que su compañera está evidentemente llamada a aventajarlo en ellas. Seamos menos vanos y egoístas, pongamos aquí a la mujer en posesión de su imperio natural. (p. 1).}

Pero la teoría va mucho más lejos y sostiene que el propio Gutiérrez es un artista de excelencia gracias a que ha logrado cultivar lo femenino en sí: la sensibilidad, la percepción, la fuerza en la ejecución, la afección. Teniendo en cuenta que el hecho de realizar una academia y un salón para mujeres resultaba un acto revolucionario, la teoría de lo femenino en el artista, su legítimo cultivo, no solo se podía tomar como inadecuada sino como una herejía, más tratándose de Pombo, un miembro del Partido Conservador colombiano. Pero no resulta extraño que una idea tan controversial naciera de la voz de Pombo, quien ya había explorado algunas posibilidades de la sensibilidad femenina en su poema Edda, y que por esta razón fuese conocido internacionalmente con este seudónimo femenino. Sin duda alguna hay algo de travestismo en esta serie de gestos en los cuales se reconoce el poderoso potencial creativo de lo femenino y las distintas luchas que está dispuesto a dar el poeta bogotano con el ánimo de encontrar una fundamentación artística en lo natural providente. En este acto se revela la innovación de la teoría femenina de la producción artística que de un lado se emparenta con la creatividad, la fertilidad, con las fuerzas creadoras de la naturaleza, pero por otra parte no se restringe exclusivamente al dominio de la mujer, al sostener que el hombre también puede cultivar dichos atributos si se lo llega a proponer. Pombo (1874, agosto 13) afirma: 
De lo dicho debe inferirse que el director de las dos Academias, señor Gutiérrez, no sería tan completo artista como es, si la caprichosa naturaleza no hubiera enriquecido su genio con ciertas dotes esencialmente femeninas. Tal vez por su parentesco con las flores, tienen las mujeres una maravillosa percepción del color, hasta en sus más desvanecidos matices; y en este don, rarísimo entre pintores, el mexicano deja muy atrás a cuantos conocemos, con las ocho o diez excepciones que nadie ignora encabezadas por el Tiziano. Las mujeres, en la batalla de la vida, compensan la osadía y la fuerza del hombre, con la observación y con su sensibilidad más que barométrica para los sentimientos ajenos. (p. 1).

Tanto para Pombo como para Gutiérrez, lo femenino pasa de ser un lugar no deseado en las academias de artes a ser una de las cualidades principales del artista "modernista", porque solo esta cualidad puede otorgarle su gravitación, vitalidad, vibración, sensibilidad en el matiz y el color. Lo femenino ofrece mayor simpatía en el color y un espectro más amplio en lo sensible, en los aspectos formales, en tanto en los filosóficos brinda una posibilidad de volver a crear vínculos sagrados entre la humanidad y la naturaleza por medio de la ampliación del espectro simbólico, ya no de dominación metafísica sino de armonía, simpatía y simbiosis en la habitación de la sobrenaturaleza americana. 


\section{REFERENCIAS BIBLIOGRÁFICAS}

Arboleda, S. (1873, junio 7). Informe sobre Bellas Artes. La Escuela Normal: periódico oficial de instrucción pública, p. 184.

Calvo Serraller, F. (1990). Pintores españoles entre dos fines de siglo (1880-1990): de Eduardo Rosales a Miquel Barceló. Madrid: Alianza.

Creación de la Academia Vásquez. (1873, junio 7). La Escuela Normal, periódico oficial de instrucción pública, Tomo IV, N 127, p. 183.

Gutiérrez, F. S. (1885). Tratado del dibujo y la pintura: con un apéndice de los diversos caracteres de las escuelas antiguas y modernas. Colima, México: Sociedad de Mejoras Materiales.

Gutiérrez, F.S. (1883) Viaje de Felipe S. Gutiérrez por México, los Estados Unidos, Europa y Sud-América. México: Tipografía literaria de Eugenio Malta.

Martí, J. (2000a). Obras Completas (Tomo IV). La Habana: Centro de Estudios Martianos.

Martí, J. (2000b). Obras Completas (Tomo III). La Habana: Centro de Estudios Martianos.

Medina, A. (2014). Procesos del arte en Colombia. Bogotá: Universidad de los Andes - Laguna Libros.

Pombo, R. (1917). Poesías (Tomo II). Bogotá: Imprenta Nacional. 
Pombo, R. (1875, junio 3). Pombo y Gutiérrez. Revista Universal. Tomado de I. Rodríguez, La crítica de arte en México en el siglo XIX: Estudios y documentos III (1879-1902). México: UNAM.

Pombo, R. (1874, agosto 13). Exposición de Bellas Artes. La América, p. 1.

Pombo, R. (1874, mayo 11). Exposición Gutiérrez. La América, p. 2.

Pombo, R. (1874, febrero 28). La educación es la fuerza de la mujer. La Escuela Normal: periódico oficial de instrucción pública, p. 64.

Pombo, R. (1873, noviembre 20). Memorial sobre la Academia Vázquez. La América, p. 1.

Pombo, R. (1873, septiembre 22). La llegada de Gutiérrez. La América, N 121, p. 2.

Tibble, C. (2016, junio 28). Bordar, pintar y tocar el piano. Bogotá, Colombia. Revista Arcadia. Recuperado de: http://www.revistaarcadia.com/ 